\title{
NEW INVARIANTS OF OPEN SYMPLECTIC AND CONTACT MANIFOLDS
}

\author{
YAKOV ELIASHBERG
}

\section{INTRODUCTION}

The first specifically symplectic invariant of open symplectic manifolds, the width, was defined by M. Gromov in [Gr]. Gromov's width was generalized by H. Hofer, I. Ekeland, E. Zehnder, and A. Floer (see [EH, HZ, FH]). They defined numerous new invariants, i.e., symplectic capacities.

All these invariants are monotonic with respect to the inclusion and serve as obstructions for symplectic embeddings. For contact manifolds, so far no invariants are known (except "overtwisting phenomenon" in dimension three (see [Be1, El1]). It turns out that much more rich obstructions for symplectic (see 2.2) and, what is especially interesting, for contact embeddings (see 3.2 and 3.5) can be defined if we consider embeddings in a prescribed homotopy class.

This paper is inspired by a recent discovery by Benci and Sikorav (see [Bc, Si1, LS], and 2.4.1 below). Recently J.-C. Sikorav (see [Si2]) independently developed some of the ideas discussed in this paper. His interests are exclusively in the symplectic field while I am interested mainly in applications to the contact topology. The most interesting result of this paper is Theorem 3.5.1, which shows, in particular, the existence of nonisomorphic open solid tori in the standard contact $S^{3}$.

\section{SyMPLECTIC MANIFOLDS}

2.1. Periods of a Lagrangian embedding. Let $(X, \omega)$ be a symplectic manifold of dimension $2 n$ and $M$ be a closed connected $n$-dimensional manifold. An embedding $f: M \rightarrow X$ is called Lagrangian if $f^{*} \omega=0$. If $\omega$ is exact then any primitive $\lambda$ with $d \lambda=\omega$ is called a Liouville form. Suppose that $\omega$ is exact (see Remark 2.2.1 below). Then for a chosen Liouville form $\lambda$ the form $f^{*} \lambda$ on $M$ is closed. Its cohomology class $\lambda_{f}=\left[f^{*} \lambda\right] \in H^{1}(M ; \mathbb{R})$ is called $\lambda$ period of the Lagrangian embedding $f: M \rightarrow X$. If one changes the Liouville form $\lambda$ by $\mu=\lambda+\theta$ with $d \theta=0$ then $\mu_{f}=\lambda_{f}+f^{*}[\theta]$ where [ $\left.\theta\right]$ is a cohomology class of $\theta$ in $H^{1}(X ; \mathbb{R})$.

Received by the editors August 24, 1990.

1980 Mathematics Subject Classification (1985 Revision). Primary 58F05, 53C15, 57R15.

Partially supported by NSF grant DMS-9006179. 
2.2. Shapes. Let $(X, \omega)$ and $M$ be as in 2.1. Let us fix a homomorphism

$$
\alpha: H^{1}(X ; \mathbb{R}) \rightarrow H^{1}(M ; \mathbb{R}) .
$$

Let $\lambda$ be a Liouville form in $X$, i.e., $d \lambda=\omega$.

Denote by $I(X \mid M, \alpha)$ the subset of $H^{1}(M ; \mathbb{R})$ that consists of points $z \in$ $H^{1}(M ; \mathbb{R})$ such that there exists a Lagrangian embedding $f: M \rightarrow X$ with $f^{*}=\alpha$ and $z=\lambda_{f}$.

As it was mentioned in 2.1 the change of a Liouville form $\lambda$ causes a translation of $I(X \mid M, \alpha)$ in $H^{1}(M ; \mathbb{R})$. So we will consider the set $I(X \mid M, \alpha)$ to be defined up to a translation. For two sets $A, B$, which are defined up to translation, the inclusion $A \subset B$ means that $A$ can be included in $B$ by a translation.

We call $I(X \mid M, \alpha)$ the $(M, \alpha)$-shape of $X$. Of course, $(M, \alpha)$-shape of $X$ can be empty but sometimes it contains a lot of information about the symplectic manifold $(X, \omega)$.

Remark 2.2.1. The definition of the shape-invariant can be extended to a nonexact symplectic manifold $(X, \omega)$. In this case the shape should be considered a subset of $H^{1}(M ; \mathbb{R} / G)$, where $G$ is a subgroup of the image of the homomorphism $[\omega]: H_{2}(X) \rightarrow \mathbb{R}$ defined by the formula $[\omega](z)=\int_{z} \omega, z \in H_{2}(X)$.

\subsection{A functorial property of the $(M, \alpha)$-shape.}

Proposition 2.3.1. Let $\left(U_{1}, \omega_{1}\right)$ and $\left(U_{2}, \omega_{2}\right)$ be two symplectic manifolds and $h: U_{1} \rightarrow U_{2}$ be a symplectic embedding. Let $M$ and the homomorphism $\alpha: H^{1}\left(U_{1} ; \mathbb{R}\right) \rightarrow H^{1}(M ; \mathbb{R})$ be as above.

Then

$$
I\left(U_{1} \mid M, \alpha\right) \subset I\left(U_{2} \mid M, \alpha \circ h^{*}\right) .
$$

Proof. Let $d \lambda_{2}=\omega_{2}$ and $\lambda_{1}=f^{*} \lambda_{2}$. For a point $a \in I\left(U_{1} \mid M, \alpha\right)$ there exists a Lagrangian embedding $f: M \rightarrow U_{1}$ such that $f^{*}=\alpha$ and $a=\left(\lambda_{1}\right)_{f}$. Then $h \circ f$ is a Lagrangian embedding $M \rightarrow U_{2}$ and $(h \circ f)^{*}=\alpha \circ h^{*}: H^{1}\left(U_{2} ; \mathbb{R}\right) \rightarrow$ $H^{1}(M ; \mathbb{R})$. Moreover, we have $(h \circ f)^{*} \lambda_{2}=f^{*} \lambda_{1}$ and, therefore, $a=\left(\lambda_{1}\right)_{f}=$ $\left(\lambda_{2}\right)_{h \circ f} \in I\left(U_{2} \mid M, \alpha \circ h^{*}\right)$.

Proposition 2.3.1 shows that the shape $I(X \mid M, \alpha)$ can serve as an obstruction to a symplectic embedding in a given homotopical class.

2.4. Shapes of certain manifolds. The theorem of this section belongs to Sikorav (see [Si2]) and is a reformulation of an earlier theorem by Benci-Sikorav (see [Bc, Si1]), which is based on Gromov's result about intersections of exact Lagrangian manifolds (see $[\mathrm{Gr}]$ ).

Let $T^{n}=\mathbb{R}^{n} / \mathbb{Z}^{n}$ and $X$ be the cotangent bundle $T^{*}\left(T^{n}\right)$ with the canonical symplectic structure $\omega=d\left(\sum_{1}^{n} p_{i} d q_{i}\right)$. Note that $X=T^{*}\left(\mathbb{R}^{n} / \mathbb{Z}^{n}\right)$ canonically decomposes into the product $\mathbb{R}^{n} / \mathbb{Z}^{n} \times \mathbb{R}^{n}$ with the symplectic structure defined by the form $d\left(\sum_{i=1}^{n} p_{i} d q_{i}\right)$ where coordinates $\left(q_{1}, \ldots, q_{n}\right)$ and $\left(p_{1}, \ldots, p_{n}\right)$ 
correspond to the first and second factor respectively. Note that tori $\{p=$ $\left(p_{1}, \ldots, p_{n}\right)=$ const $\} \subset X$, which are graphs of closed forms $\sum_{1}^{n} p_{i} d q_{i}$ on $T^{n}$, are Lagrangian. Choosing cohomology classes $\left[d q_{1}\right], \ldots,\left[d q_{n}\right]$ as a basis of $H^{1}\left(T^{n} ; \mathbb{R}\right)$ we identify $H^{1}\left(T^{n} ; \mathbb{R}\right)$ with the fiber $\mathbb{R}^{n}$ of the fibration $T^{*}\left(T^{n}\right) \rightarrow$ $T^{n}$. The cohomology class of a form $\sum_{1}^{n} p_{i} d q_{i}$ corresponds to the point $p=$ $\left(p_{1}, \ldots, p_{n}\right) \in \mathbb{R}^{n}$ under this identification.

Let $i$ be the inclusion

$$
T^{n}=T^{n} \times 0 \hookrightarrow T^{*}\left(T^{n}\right) .
$$

Theorem 2.4.1. Let $A \subset \mathbb{R}^{n}$ be a connected open subset and $U=T^{n} \times A \subset$ $T^{*}\left(T^{n}\right)$. Then $\left(T^{n}, i^{*}\right)$-shape of $U$ is equal to $A$.

\section{Contact MANifoldS}

3.1. Symplectization. Let $U$ be a $(2 n-1)$-dimensional manifold. A contact structure $\xi$ on $U$ is a completely nonintegrable codimension 1 tangent distribution. It means that $\xi$ can be defined, at least locally, by a 1 -form $\lambda$ with $\lambda \wedge(d \lambda)^{n-1} \neq 0$. Note that if $n$ is odd then the contact distribution $\xi$ is automatically orientable. For an even $n$ the existence of a contact structure implies the orientability of the ambient manifold $U$. In both cases, the coorientability of $\xi$ implies that $\xi$ and $U$ are both orientable. We will assume from now on that $\xi$ is coorientable and fix its coorientation. Then $\xi$ can be globally defined by a 1 -form $\lambda$, which is determined up to a multiplication by a positive function. Let $\mathscr{S} U=U \times] 0,+\infty[$. We will still denote by $\lambda$ the pull-back of $\lambda$ by the projection of $\mathscr{S} U=U \times \mathbb{R}_{+}$on the first factor and denote by $t$ the projection on the second. Then the form $\omega=d(t \lambda)$ defines a symplectic structure on $\mathscr{S} U$ (indeed, $(d(t \lambda))^{n}=t^{n-1} d t \wedge \lambda \wedge d \lambda^{n-1} \neq 0$ ). The map $(x, t) \longmapsto(x, t / f(x))$ induces an isomorphism of forms $d(t \lambda)$ and $d(t \mu)$ for $\mu=f \lambda$. Therefore, the symplectic manifold $(\mathscr{S} U, \omega)$ depends, up to a symplectomorphism, only on the contact manifold $(U, \xi)$ and not on the choice of the 1 -form $\lambda$. We will denote $(\mathscr{S} U, \omega)$ by $\operatorname{Sympl}(U, \xi)$ and call it the symplectization of the contact manifold $(U, \xi)$ (comp. [A, El2]). Note that a contact embedding $f:\left(U_{1}, \xi_{1}\right) \rightarrow\left(U_{2}, \xi_{2}\right)$ induces a $\mathbb{R}_{+}$-equivariant symplectic embedding,

$$
\operatorname{Sympl}(f): \operatorname{Sympl}\left(U_{1}, \xi_{1}\right) \rightarrow \operatorname{Sympl}\left(U_{2}, \xi_{2}\right) .
$$

Therefore, "Sympl" can be considered a functor from the category of contact manifolds and their contact maps to the category of symplectic manifolds with $\mathbb{R}_{+}$- action and their $\mathbb{R}_{+}$-equivariant (homogeneous) symplectic maps.

Note that the symplectic manifold $(\mathscr{S} U, \omega)$ is exact and the condition of equivariancy fixes the Liouville form on $S U$. In other words, equivariant symplectomorphisms of ( $S U, \omega=d(t \lambda)$ ) leaves invariant the Liouville form $t \lambda$.

Examples 3.1.1. (a) For an $n$-dimensional manifold $M$ let us denote by $S T^{*}(M)$ the unit cotangent bundle of $M$ with the contact structure $\xi$ defined by the 
restriction of the form $p d q$. The manifold $S T^{*} M$ can also be considered a space of cooriented $(n-1)$-dimensional contact elements of $M$. With this interpretation the plane $\xi_{x}$ of $\xi$ at a point $x=(p, q), q \in M, p \in T_{q}^{*}(M)$, consists of infinitesimal deformations of $\xi_{x}$, which leaves fixed the point of contact $q \in M$. Then the symplectization $\operatorname{Sympl}\left(S T^{*}(M), \xi\right)$ is isomorphic to $T^{*}(M) \backslash M$ with the standard symplectic structure $\omega=d(p d q)$.

(b) The standard contact structure $\xi_{0}$ on the unit sphere $S^{2 n-1} \subset \mathbb{C}^{n}$ is formed by complex tangencies to it, i.e., by $(2 n-2)$-dimensional tangent hyperplanes perpendicular to fibers of the Hopf fibration. The symplectization of $\left(S^{2 n-1}, \xi_{0}\right)$ is $\mathbb{C}^{n} \backslash 0$ with the standard symplectic structure. For any domain $U \subset S^{2 n-1}$ the symplectization $\operatorname{Sympl}\left(U, \xi_{0}\right)$ is a cone over $U$ in $\mathbb{C}^{n}$ without its vertex $0 \in \mathbb{C}^{n}$, with the standard symplectic structure induced from $\mathbb{C}^{n}$.

It is a natural idea to try to define contact invariants of the contact manifold $(U, \xi)$ through symplectic invariants of its symplectization $\operatorname{Sympl}(U, \xi)$. Unfortunately, all known symplectic invariants (like volume, width, symplectic capacities) are infinite for $\operatorname{Sympl}(U, \xi)$ and, therefore, do not give any information about the contact manifold. The situation is completely different with the shape-invariant as we show it in the rest of the paper.

3.2. Shape-invariant in the contact case. Let $(U, \xi)$ be a contact manifold as in 3.1 and $M$ be an $n$-dimensional connected closed manifold. Let us fix a homomorphism $\alpha: H^{1}(U ; \mathbb{R}) \rightarrow H^{1}(M ; \mathbb{R})$. If $(\mathscr{S} U, \omega)$ is the symplectization of $(U, \xi)$ then $H^{1}(\mathscr{S} U ; \mathbb{R})$ is canonically isomorphic to $H^{1}(U ; \mathbb{R})$ and, in particular, $\alpha$ can be considered an isomorphism $H^{1}(\mathscr{S} U ; \mathbb{R}) \rightarrow H^{1}(M ; \mathbb{R})$. Then the symplectic shape $I(\mathscr{S} U \mid M, \alpha)$ is an invariant of the contact manifold $(M, \xi)$ as it follows from the discussion in 3.1. Moreover, the symplectization of a contactomorphism $(U, \xi) \rightarrow(U, \xi)$ preserves not only $\omega=d(t \lambda)$ but the Liouville form $t \lambda$ itself (see 3.1). Therefore, the shape $I(\mathscr{S} U \mid M, \alpha)$ as a contact invariant can be defined without any freedom of translation.

Finally note that $I(\mathscr{S} U \mid M, \alpha) \subset H^{1}(U ; \mathbb{R})$ is a cone (without the vertex). Therefore, it is more convenient to projectivize* the invariant and consider the set $P I(\mathscr{S} U \mid M, \alpha)=I(\mathscr{S} U \mid M, \alpha) / \mathbb{R}_{+} \subset P H^{1}(U ; \mathbb{R})$.

Let us summarize the above discussion in the following

Definition 3.2.1. Let $(\mathscr{S} U, \omega)=d(t \lambda)$ be the symplectization of a contact manifold $(U, \xi), M$ be an $n$-dimensional closed connected manifold and $\alpha: H^{1}(U ; \mathbb{R}) \rightarrow H^{1}(M ; \mathbb{R})$ be a homomorphism. Let $A, A \subset H^{1}(M ; \mathbb{R})$, be the set of all cohomology classes from $H^{1}(M ; \mathbb{R})$ that can be represented by a closed form $\left[f^{*}(t \lambda)\right]$ for a Lagrangian embedding $f: M \rightarrow \mathscr{S} U$ with $f^{*}=\alpha$. Then the contact $(M, \alpha)$-shape of $(U, \xi)$ is the projectivization of $A$,

*In this paper I use the word "projectivization" in the sense of identification of vectors that differ by a positive factor. Therefore, the projectivization $P E$ of a vector space $E$ is a sphere rather than a projective space. The projectivization of homomorphisms are understood in the same sense. 


$$
I_{C}(U \mid M, \alpha)=P A \subset P H^{1}(M ; \mathbb{R}) .
$$

The following Proposition 3.2.2 is a contact version of 2.3.1 and its proof, as in the symplectic case, is straightforward.

Proposition 3.2.2. Let $h:\left(U_{1}, \xi_{1}\right) \rightarrow\left(U_{2}, \xi_{2}\right)$ be a contact embedding $\left(\operatorname{dim} U_{1}=\operatorname{dim} U_{2}=2 n-1\right), M$ be an n-dimensional connected closed manifold and $\alpha$ be a homomorphism $H^{1}\left(U_{1}, \mathbb{R}\right) \rightarrow H^{1}(M ; \mathbb{R})$. Then

$$
I_{C}\left(U_{1} \mid M, \alpha\right) \subset I_{C}\left(U_{2} \mid M, \alpha \circ h^{*}\right) \text {. }
$$

3.3. Invariant $\widetilde{I}_{C}$. In this section we give another version of the definition of the contact shape. The new definition has an advantage to be formulated in intrinsic contact terms without going to the symplectization.

An embedding $\varphi: M \rightarrow U$ where $M, U$, as in 3.2., is called coisotropic if the following two conditions are fulfilled:

$-\varphi(M)$ is transversal to the contact structure $\xi$;

- The (codimension 1) distribution $\varphi^{*}(\xi) \subset T(M)$ is integrable and can be defined by a closed smooth 1 -form.

Note that the last condition can be equivalently expressed by saying that the foliation defined by the distribution $\varphi^{*}(\xi)$ admits a smooth transversal measure.

Fix a homomorphism $\alpha: H^{1}(U ; \mathbb{R}) \rightarrow H^{1}(M ; \mathbb{R})$ and let $\widetilde{I}_{C}(U \mid M, \alpha)=$ $\left\{\left[\varphi^{*}(\lambda)\right]\right.$, where $\varphi$ is a coisotropic embedding $M \rightarrow U$, with $\varphi^{*}=\alpha$, the 1 -form $\lambda$ defines $\xi$ and the form $\varphi^{*} \lambda$ is closed $\} \subset H^{1}(M ; \mathbb{R})$. We will call $\widetilde{I}_{C}(U \mid M, \alpha)$ the modified contact $(M, \alpha)$-shape of $U$.

The relationship between the shapes is described in Proposition 3.3.1 below. The construction of a Lagrangian lift, which I use there, is due to D. Bennequin [Be2].

\section{Proposition 3.3.1.}

$$
\widetilde{I}_{C}(U \mid M, \alpha) \subset I_{C}(U \mid M, \alpha) .
$$

Proof. Let $\lambda$ be a 1 -form that defines $\xi$ and $\varphi: M \rightarrow U$ be a coistropical embedding. Suppose that for a positive function $f: M \rightarrow \mathbb{R}$ the form $\varphi^{*}(f \lambda)$ is closed. Then the embedding $\Phi: x \longmapsto(\varphi(x), 1 / f(x))$ of $M$ into $\mathscr{S} U=$ $U \times \mathbb{R}_{+}$is, evidently, Lagrangian and $\Phi^{*}(t \lambda)=\varphi^{*}(\lambda)$.

Remark 3.3.2. In all examples I know we have $I_{C}=\widetilde{I}_{C}$.

3.4. Computation of the contact shape. In this section we reformulate Theorem 2.4.1 for the contact case.

Let me remind that the unit cotangent bundle $S T^{*}\left(T^{n}\right)$ carries a canonical contact structure $\xi$ defined by the 1 -form $p d q$ (see 3.1.1). The decomposition $T^{*}\left(T^{n}\right)=T^{n} \times \mathbb{R}^{n}$ described in 2.4 gives the decomposition $S T^{*}\left(T^{n}\right)=$ $T^{n} \times S^{n-1}$ and the identification from 2.4 of $H^{1}\left(T^{n} ; \mathbb{R}\right)$ with the fiber $\mathbb{R}^{n}$ of the fibration $T^{n} \times \mathbb{R}^{n} \rightarrow T^{n}$ gives an identification of the projectivized group $P H^{1}\left(T^{n} ; \mathbb{R}\right)$ with the fiber $S^{n-1}$ of the fibration $S T^{*}\left(T^{n}\right)=T^{n} \times S^{n-1} \rightarrow T^{n}$. 
Proposition 3.4.1. Let $A \subset S^{n-1}$ be a connected open subset and $i$ be an embedding $x \longmapsto(x, a) ; a \in A$. Then

$$
I_{C}\left(T^{n} \times A \mid T^{n}, i^{*}\right)=A .
$$

Proof. The symplectization of $T^{n} \times A$ equals $T^{n} \times C A$, where $C A=\{t x \in$ $\left.\mathbb{R}^{n} \mid t \in \mathbb{R}_{+}, x \in A\right\}$ (see 3.1.1). So, applying 2.3.1 we get $I\left(T^{n} \times C A \mid T^{n}, i^{*}\right.$ ) $=C A$. Projectivizing the result we get $I_{C}\left(T^{n} \times A \mid T^{n}, i^{*}\right)=A$.

In the next section Proposition 3.4.1 is applied to get the classification of round open 3-dimensional solid tori in the standard contact $S^{3}$.

3.5. Classification of 3-dimensional solid tori. Let $\left(S^{3}, \xi\right)$ be the standard contact sphere, i.e., $\xi$ is a field of complex tangencies to the unit sphere $S^{3} \subset \mathbb{C}^{2}$. Let $\left(z_{1}, z_{2}\right)$ be a coordinate system in $\mathbb{C}^{2}$. For a positive $\delta<1$ let us denote by $U_{\delta}$ the open solid torus $\left\{\left|z_{1}\right|<\delta\right\} \cap S^{3}$.

Theorem 3.5.1. $U_{\delta}$ is contactomorphic to $U_{\delta^{\prime}}$ if and only if the difference $1 / \delta^{2}-$ $1 /\left(\delta^{\prime}\right)^{2}$ is an integer.

Proof. Let $z_{k}=\rho_{k} e^{i \varphi_{k}}, k=1,2$. Then the contact structure $\xi$ on $S^{3}$ can be defined by the 1 -form

$$
\alpha=\rho_{1}^{2} d \varphi_{1}+\rho_{2}^{2} d \varphi_{2} \quad \text { where } \rho_{1}^{2}+\rho_{2}^{2}=1 .
$$

First note that if $1 / \delta^{2}-1 /\left(\delta^{\prime}\right)^{2}=-k, k>0$, then the map

$$
\left(\rho_{1}, \varphi_{1}, \varphi_{2}\right) \longmapsto\left(\frac{\rho_{1}}{\sqrt{1+k \rho_{1}^{2}}}, \varphi_{1}-k \varphi_{2}, \varphi_{2}\right)
$$

is contact and sends $U_{\delta}$ onto $U_{\delta^{\prime}}$. Suppose now that there exists a contactomorphism $\Phi: U_{\delta} \rightarrow U_{\delta^{\prime}}$. For $0<\alpha<\beta<1$ let us denote by $V_{\alpha \beta}$ the domain $\left\{\alpha<\rho_{1}<\beta\right\} \subset S^{3}$. Let $S T^{*}\left(T^{2}\right)$ be the unit cotangent bundle of the torus $T^{2}$ and $S T^{*}\left(T^{2}\right)=T^{2} \times S^{1}$ be its canonical decomposition (see 3.4). The standard contact structure on $T^{2} \times S^{1}$ can be defined by a 1 -form $\gamma=\cos \theta d \varphi_{1}+\sin \theta d \varphi_{2}$ where cyclic coordinates $\left(\varphi_{1}, \varphi_{2}\right)$ and $\theta$ correspond to the two factors.

Let $\Gamma_{\theta_{1} \theta_{2}}, \Gamma_{\theta_{1} \theta_{2}} \subset S^{1}$ be the arc $\theta_{1}<\theta<\theta_{2}$. Then the map

$$
g:\left(\rho_{1}, \varphi_{1}, \varphi_{2}\right) \longmapsto\left(\arctan \left(\frac{1}{\rho_{1}^{2}}-1\right), \varphi_{1}, \varphi_{2}\right)
$$

is a contact embedding of $S^{3} \backslash\left\{z_{1} z_{2}=0\right\}$ in $S T^{*}\left(T^{2}\right)$ and

$$
g\left(V_{\alpha \beta}\right)=T^{2} \times \Gamma_{h(\alpha) h(\beta)} \subset T^{2} \times S^{1}=S T^{*}\left(T^{2}\right),
$$

where $h(t)=1 / t^{2}-1,0<t<1$. Now we have (see 3.2.2)

$$
I_{C}\left(V_{\varepsilon \delta} \mid T^{2}, i^{*}\right) \subset I_{C}\left(V_{\varepsilon^{\prime} \delta^{\prime}} \mid T^{2}, i^{*} \circ \Phi^{*}\right) .
$$


There exists an isomorphism $\psi: H^{1}\left(T^{2} ; \mathbb{R}\right) \rightarrow H^{1}\left(T^{2} ; \mathbb{R}\right)$ such that $\psi \circ i^{*}=$ $i^{*} \circ \Phi^{*}$. Let $P \psi: P H^{1}\left(T^{2} ; \mathbb{R}\right) \rightarrow P H^{1}\left(T^{2} ; \mathbb{R}\right)$ be the projectivization of $\psi$. Then

$$
I_{C}\left(V_{\varepsilon^{\prime} \delta^{\prime}} \mid T^{2}, i^{*} \circ \Phi^{*}\right)=P \psi\left(I_{C}\left(V_{\varepsilon^{\prime} \delta^{\prime}} \mid T^{2}, i^{*}\right)\right) \text {. }
$$

Note that $\Phi$ is defined on the whole solid torus $U_{\delta}$ and, therefore, $\Phi^{*}$ and $\psi$ preserve the cohomology class $\left[d \varphi_{2}\right]$. Therefore, the automorphism $\psi$ has the matrix

$$
A=\left(\begin{array}{ll}
1 & 0 \\
k & 1
\end{array}\right)
$$

in the basis $\left[d \varphi_{1}\right],\left[d \varphi_{2}\right]$. It is important that the integer $k$ depends only on $\Phi$ and not on the choice of $\varepsilon^{\prime}$.

Now identifying $V_{\alpha \beta}$ with $T^{2} \times \Gamma_{h(\alpha) h(\beta)}$ and using 3.4.1 we have

$$
I_{C}\left(V_{\alpha \beta} \mid T^{2}, i^{*}\right)=\Gamma_{h(\alpha) h(\beta)} .
$$

Therefore,

$$
\Gamma_{h(\varepsilon) h(\delta)} \subset P \psi\left(\Gamma_{h\left(\varepsilon^{\prime}\right) h\left(\delta^{\prime}\right)}\right) .
$$

But $P \psi\left(\Gamma_{x y}\right)=\Gamma_{x+k, y+k}$ for any $0<x<y$. Therefore,

$$
h\left(\varepsilon^{\prime}\right)+k \geq h(\delta) \geq h\left(\delta^{\prime}\right)+k
$$

and for $\varepsilon^{\prime} \rightarrow \delta$ we get

$$
h(\delta)=h\left(\delta^{\prime}\right)+k
$$

or

$$
1 / \delta^{2}-1 /\left(\delta^{\prime}\right)^{2}=k
$$

\section{ACKNOWLEDGMENTS}

The paper was finished while I visited ETH-Zentrum in Zürich. I want to thank the Forschungsinstitut für Mathematik for the hospitality.

\section{REFERENCES}

[A] V. I. Arnold, Mathematical methods of classical mechanics, MIR, Moscow, 1974.

[Bc] V. Benci, Talk at the Conference in Symplectic Geometry, MSRI, Berkeley, 1988.

[Be1] D. Bennequin, Entrelacement et equation de Pfaff, Astérisque 107-108 (1983), 87-161.

[Be2] _ private communication, 1989.

[EH] I. Ekeland and H. Hofer, Symplectic topology and Hamiltonian dynamics. I, Math. Z. 200 (1989), 355-378; II. preprint, 1989.

[E11] Ya. Eliashberg, Classification of overtwisted contact structures on 3-manifolds, Invent. Math. 98 (1989), 623-637.

[E12] _ Three lectures on symplectic topology, Cala Gonone, 1988.

[FH] A. Floer and H. Hofer, in preparation.

[Gr] M. Gromov, Pseudo-holomorphic curves in symplectic manifolds, Invent. Math. 82 (1985), 460-472.

[HZ] H. Hofer and E. Zehnder, A new capacity for symplectic manifolds, Analysis Et Cetera, Academic Press, Boston, 1990. 
[LS] F. Lalonde and J.-C. Sikorav, Sous-variétés exactes des fibrés cotangent, preprint, 1990.

[Si1] J.-C. Sikorav, Rigidité symplectique dans le cotangent de $T^{n}$, Duke Math. J. 59 (1989), 227-231.

[Si2] _ Quelques propriétés des plongement Lagrangiens, preprint, 1990.

Department of Mathematics, Stanford University, Stanford, California 94305-2125 